\title{
Comets and the Origin of Life: Structure of Comets Nuclei and Dust
}

\author{
A.Chantal Levasseur-Regourd \\ Université Paris VI / Aéronomie, BP 3, F-91371 Verrières, France
}

Emmanuel Desvoivres

Laboratoire de Planétologie de Grenoble, BP 53, F-38041, Grenoble, France

\begin{abstract}
From observations of fragmentation events, clues to a gravitational aggregates structure of some comets nuclei are given. From in-situ and remote light scattering observations, a very high porosity is estimated for dust in comae. These results suggest a possible survival of cometary organics during atmospheric entry at the epoch of intense bombardment. In the coming years, new missions to comets and laboratory measurements on the ISS, will be used to assess such a scenario.
\end{abstract}

\section{Introduction}

Past in-situ cometary missions and remote observations of bright comets indicate that comets nuclei are built up of ices and dust, rich in complex organic molecules. These molecules, with possibly amino-acids, were most likely formed on interstellar dust grains (see Keller, these proceedings). During the epoch of intense bombardment, a huge number of comets have plunged into the inner solar system, released dust in the interplanetary medium, and impacted the terrestrial planets. If the organic molecules were able to survive the atmospheric impact of the nuclei (or of the dust particles previously released), they have most likely enriched the planets in organic matter. We present evidence for the structure of some comets nuclei and cometary dust, and compare the results with those obtained for asteroids and meteoroids. Future experiments allowing better understanding of the structure of cometary nuclei and dust are then shortly presented.

\section{Structure of Comets Nuclei}

Fragmentation events, leading to the formation of bright mini-comae around a fragment, are frequently observed. The tidal disruption of D/Shoemaker-Levy 9 at Jupiter periapsis has been extensively documented in 1993-1994. A possible fragment of $26 \mathrm{P} /$ Grigg-Skjellerup has been suspected in its inner coma during the Giotto spacecraft flyby in 1992 (McBride et al. 1997).

More recently, we have monitored the evolution of mini-comae in the coma of C/1996 B2 Hyakutake. The study of the motion of the icy fragments leads to 


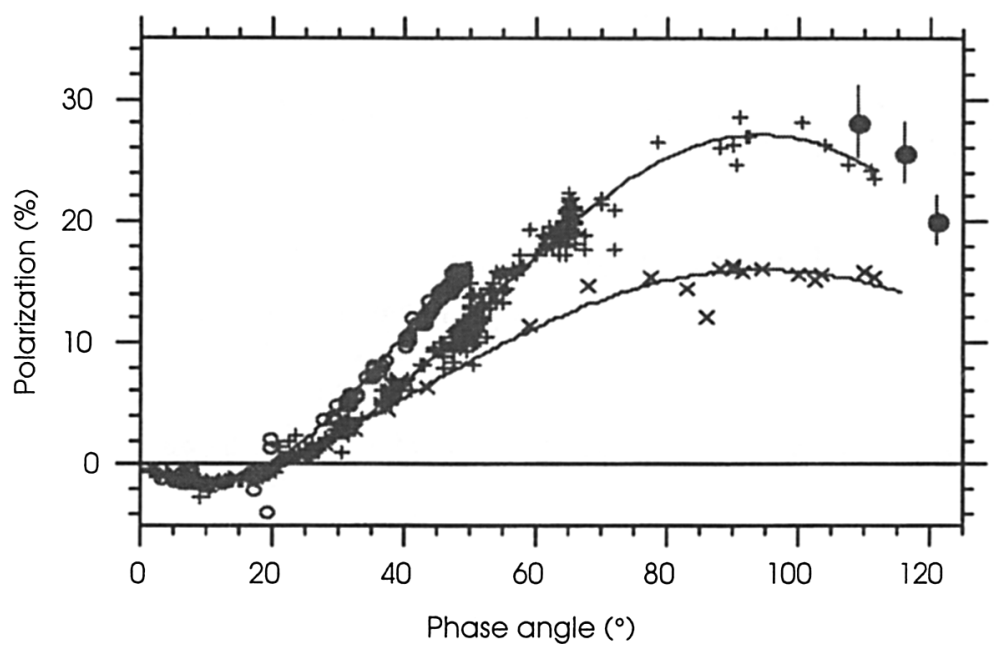

Figure 1. Polarization $\mathrm{P}$ as a function of the phase angle in the red domain. (०) C/1995 O1 Hale-Bopp; (•) LINEAR 1999 S4 at its disruption; $(\times)$, low $\mathrm{P}_{\max }$ comets; $(+)$, high $\mathrm{P}_{\max }$ comets; (LevasseurRegourd et al. 2002).

the determination of the product of their size by their density. The biggest one has a size of about 60 to $480 \mathrm{~m}$ (corresponding to a density ranging from 0.1 to $0.8 \mathrm{~g} . \mathrm{cm}^{-3}$ ), while smallest fragments may have sizes below $3 \mathrm{~m}$ (Desvoivres et al. 2000). We have also observed the disruption of C/1999 S4 LINEAR at the end of July 2000. From observations of the polarization of the solar scattered light (Fig. 1), the presence of large and rather compact icy agglomerates is suspected in the coma.

Fragmentation and total disruption of comets nuclei are rather frequent phenomena. Some nuclei could be quite fragile gravitational aggregates, built up of smaller cometesimals with very low tensile strength. Such structures could be the result of the formation processes and of mutual collisions during their lifetime.

\section{Structure of Cometary Dust}

A remarkable agreement has been found, from Giotto in-situ observations, between the dust light scattering (OPE) and impact (DID) data sets (LevasseurRegourd et al. 1999). The derived model suggests that the dust particles are dark and porous, the best fit corresponding to values of the density and the albedo respectively equal to $0.1 \mathrm{~g} . \mathrm{cm}^{-3}$ and 0.04 (Fulle et al. 2000).

Remote observations of the dust light scattering properties (linear polarization $\mathrm{P}$ ) lead to a smooth dependence of $\mathrm{P}$ on the phase angle (see Figure 1, for large aperture observations), and to an increase of $\mathrm{P}$ (at a given phase angle) with increasing wavelength (Levasseur-Regourd 1999). Such results give clues to the 
structure of the dust. Light scattering measurements on clouds of dust particles levitating in micro-gravity conditions actually indicate that similar variations of $\mathrm{P}$ with phase angle and wavelength are obtained for extremely porous aggregates of sub-micron sized dust particles (Worms et al. 1999; Hadamcik et al. 2002).

These results agree with dust clouds experiments reproducing the earliest stage of planetary formation. Low velocity collisions experiments actually produce agglomerates with a fractal dimension of about 1.3 (Blum et al. 2000).

\section{Discussion and conclusions}

The density of asteroids, when available, seems to be lower than that of their meteorite analogues, and it is estimated that about $16 \%$ of the NEO (Near Earth asteroids) with sizes above $200 \mathrm{~m}$ are binary objects (Margot et al. 2002). Some asteroids could thus be gravitational aggregates; these objects being slow rotators, as opposed to monolithic faster rotating asteroids with sizes below about $100 \mathrm{~m}$. Although all comets are certainly not identical, it may now be assumed that some cometary nuclei are gravitational aggregates. Atmospheric cometary impacts have been modelled for sizes above $2 \mathrm{~km}$ (Pierazzo and Chyba, 1999). Since the survival of amino-acids in comets decreases with impacting body size (Pierazzo, these proceedings), it would be of interest to test models for the $10 \mathrm{~m}-1 \mathrm{~km}$ size range.

Porous IDPs (Interplanetary Dust Particles) have been collected in the stratosphere (Jessberger et al. 2001). Although the density of meteoroids is rather difficult to determine $\left(0.4 \mathrm{~g} . \mathrm{cm}^{-3}\right.$ for the Leonids, Babadzhanov 2002), and the detection of organic matter deposition in the atmosphere is yet to be done (Jenniskens, these proceedings), it may be estimated that cometary dust, which is rich in organic compounds, is built of highly porous dust aggregates, possibly embedded with ices.

From our present understanding, comets could thus have contributed, shortly after the terrestrial planets formation, to the delivery on Earth of carbonbased molecules on which life is based.

\section{Prospective}

Amongst the future missions devoted to small bodies exploration, NASA Deep Impact and ESA Rosetta should provide key information about the structural properties of comets. Deep Impact will throw a $370 \mathrm{~kg}$ copper projectile on the nucleus of $9 \mathrm{P} /$ Tempel 1 , and thus investigate its tensile strength. Rosetta will rendezvous during three years with comet $46 \mathrm{P} /$ Wirtanen and land a module on its nucleus; the structure of the dust particles (and their evolution along the comet's orbit) should be provided by MIDAS experiment, while CONSERT experiment should determine the structure of the nucleus and the size of the cometesimals.

Laboratory measurements on dust particles levitating in micro-gravity conditions, during parabolic flight campaigns (PROGRA ${ }^{2}$, Worms et al. 1999) and rocket flight (CODAG, Levasseur-Regourd et al. 2001), have demonstrated the possibility of performing light scattering measurements on clouds and of obtaining Brownian driven agglomeration. The CODAG-2 on ESA MASER 10 rocket 
will follow the dust aggregation at ballistic velocities and monitor the scattering properties in three wavelengths. These experiments are precursors for the ESA ICAPS (Interactions in Cosmic and Atmospheric Particle Systems) facility on the International Space Station. Amongst the numerous scientific objectives of ICAPS, following the mechanism of agglomeration at the early stage of planetary formation, from micron-sized to macroscopic dusty bodies, will be of major importance for a better understanding of the structure of comets nuclei and dust.

\section{References}

Babadzhanov, P. B. 2002, A\&A, 384, 317

Blum, J. 2000, Phys.Rev.Lett, 85, 2426

Desvoivres, E., Klinger, J., \& Levasseur-Regourd, A. C. 2000, Icarus, 144, 172

Fulle, M. Levasseur-Regourd, A. C., McBride, N., \& Hadamcik, E. 2000, AJ, 119,1968

Hadamcik, E., Renard, J. B., Worms, J. C., Levasseur-Regourd, A. C., \& Masson, M. Icarus, 155,497

Jessberger, E. K., Stephan, T., Rost, D., Arndt, P., Maetz, M., Stadermann, F. J., Brownlee, D. E., Bradley, J. P., \& Kurat, G. 2001, in Interplanetary Dust, ed. E. Gruen, et al. (Springer: Heidelberg), 253

Levasseur-Regourd, A. C. 1999, Space Sci. Rev., 90, 163

Levasseur-Regourd, A. C., McBride, N., Hadamcik, E., \& Fulle, M. 1999, A\&A, 348,636

Levasseur-Regourd, A. C., Haudebourg, V., Cabane, M., \& Worms, J. C. 2001, in ESA SP-454, First International Symposium on Microgravity Research and Applications, 797

Levasseur-Regourd, A. C., Hadamcik, E., \& Gaulme, P. ESA SP-500, ACM 2002 , in press

Margot, J. L., Nolan, M. C., Benner, L. A. M., Ostro, S. J., Jurgens, R. F., Giorgini, J. D., Slade, M. A., \& Campbell, D. B. 2002, Science 296, 1445

McBride, N., Green, S., Levasseur-Regourd, A. C., Goidet-Devel, B., \& Renard, J. B. 1997, MNRAS, 289, 535

Pierazzo E., \& Chyba, C. F. 1999, Meteoritics Planet. Sci. 34, 909

Worms, J. C., Renard, J. B., Hadamcik, E., Levasseur-Regourd, A. C., \& Gayet, J. F. 1999, Icarus , 142, 281 